\title{
Lectura Dantis: Paradiso XXXI
}

\author{
Rachel Jacoff \\ Wellesley College \\ rjacoff@wellesley.edu
}

\begin{abstract}
Questo articolo analizza la struttura e il ruolo di Paradiso XXXI, il secondo dei quattro canti finali del Paradiso, tutti ambientati nell' Empireo. Il canto amplia la presentazione iniziale dell' Empireo in Canto XXX , sia con il ritratto del ruolo degli angeli che con la presentazione eterodossa dei beati nei corpi gloriosi così come appariranno nel giorno del Giudizio Universale. Il canto contiene la sostituzione sorprendente di San Bernardo con Beatrice e la preghiera di addio che Dante indirizza alla donna. Nella lettura delle similitudini del canto, la centralità dell' idea del pellegrinaggio diventa chiara, indicando il modo in cui Dante crea una poetica alternativa personale al Giubileo ufficiale di Papa Bonifacio nel 1300, l'anno stesso del poema dantesco.
\end{abstract}

Parole chiave: Dante; Empireo; angeli; Roma; Beatrice; San Bernardo; Veronica; pelligrinaggio.

\begin{abstract}
This article analyzes the structure and role of Paradiso XXXI, the second of the four final cantos of Paradiso, all set in the Empyrean. The canto amplifies the initial presentation of the Empyrean in Canto XXX, both in its depiction of the role of the angels and its heterodox presentation of the blessed in their "glorious bodies» as they will appear at the Last Judgment. It also contains the surprising substitution of Saint Bernard for Beatrice and Dante's farewell prayer to Beatrice. In reading the canto's similes, the centrality of the idea of pilgrimage becomes clear, and points to the way in which Dante creates a poetic personal alternative to Pope Boniface's Jubilee of 1300, the fictional date of Dante's poem.
\end{abstract}

Keywords: Dante; Empyrean; angels; Rome; Beatrice; St. Bernard; Veronica; pilgrimage.

Sono molto contenta di tornare a Barcellona e d'avere l'opportunità di rivedere le persone che ho incontrato quando sono stata qui quasi due anni fa. Per me è sempre un piacere partecipare a una serie di Lecturae Dantis, una tradizione secolare che continua a divulgare le opere del grande poeta fiorentino e 
che assicura che tutti i canti della Commedia ricevano la nostra attenzione. Sebbene l'Inferno rimanga la cantica più letta e più conosciuta, per me il Paradiso è più affascinante. Stasera parleremo del trentunesimo canto del Paradiso, il secondo dei canti dell'Empireo. Nei quattro canti finali del Paradiso, Dante arriva alla fine del suo viaggio, passando dall'universo materiale a quello spirituale, il cielo di pura luce: «luce intellettual, piena d'amore, amor di vero ben, pien di letizia, letizia che trascende ogni dolzore». Benché la parola «empireo" ricorra solo una volta nella Commedia (e nell' Inferno), l'idea è discussa da Dante nel Convivio dove dice: «li cattolici pongono lo cielo Empireo, che è a dire cielo di fiamma o vero luminoso. [...] Questo loco è dei spiriti beati, secondo che la Santa chiesa vuole che non può dire menzogna»(3.2). L'Empireo di Dante, diversamente da quello dei teologi scolastici, è immateriale, fuori dello spazio e fuori del tempo. Come disse Etienne Gilson, è un mito dal punto di vista fisico e una metafora dal punto di vista teologico. Identificato con la mente di Dio e con la visione beatifica degli angeli e dei santi, è il luogo dell'amore, della gioia, e della dolcezza. ${ }^{1}$

Paradossalmente, questo luogo incorporeo è il posto dove appaiono i corpi gloriosi dei beati, gli unici corpi «veri» che Dante vede nel suo viaggio. Anna Maria Chiavacci Leonardi ha giustamente detto che «questo luogo, che non ha realtà se non metafisica e mistica ci rivela infine il luogo più reale di tutta la cantica». ${ }^{2}$ La poetica dell' Empireo, un sito di paradosso e di ossimoro, è una sfida per il poeta e anche per il lettore. Dobbiamo imparare a tener insieme cose che di solito teniamo separate. La verità poetica, come quella teologica, è sopranaturale.

Ciascuno dei quattro canti finali ha un tono e uno scopo un po' diverso, e ciascuno rallenta e allo stesso tempo accelera il movimento inesorabile verso la visione finale che è l'apice del poema. Nel trentesimo canto la bella vista della fiumana di luce, con le rive "dipinte con mirabile primavera» e le "faville vive» che escono dalla fiumana per infiorarsi, — tutta questa scena viene svelata come un «umbrifero prefazio» della realtà. Dopo un battesimo oculare nella fiumana di luce le faville ed i fiori sono smascherati e Dante vede le due schiere, gli angeli e i beati, e i beati nella forma corporale che avranno dopo l'ultimo giudizio. Dante descrive l'enorme anfiteatro nel quale i beati sono posti come un fiore e anche come una città, una «rosa sempiterna» e «la nostra città». In questo "convento delle bianche stole» le bianche stole dei beati costituiscono i petali di un «colosseo floreale». ${ }^{3}$ Così Dante fonde le due idee tradizionali di paradiso come giardino e come città.

1. Citato da Christian Moevs, The Metaphysics of Dante's "Comedy", New York: Oxford University Press, 2005, p. 23.

2. Anna Maria Chiavacci Leonardi, «Introduzione», Dante Alighieri Commedia, vol. III, Milano: Mondadori, 1977, p. xxvii.

3. Prendo la frase da Peter S. Hawkins, "Are You Here?': Surprise in the Commedid", in Sparks and Seeds: Medieval Literature and its Afterlife, Essays in Honor of John Freccero, ed. Dana A. Stewart e Alison Cornish, Turnhout: Brepols, 2000, p. 182. 
La grande rosa è stata legata ai rosoni delle cattedrali dell'epoca, in particolare a San Zeno a Verona, una città dove Dante ha vissuto. La parola «rosone» non è stata usata fino a più tardi, ma la forma di quelle grandi finestre circolari suggerisce il fiore, e molte di loro erano dedicate alla Vergine. Il legame fra la Vergine e la rosa era tradizionale nella liturgia ed altrove. Ricordiamo che Dante l'ha chiamato "la rosa in che 'l verbo divino / carne si fece» nel ventitreesimo canto del Paradiso. La rosa ha così tante connotazioni religiose e secolari che è difficile delimitare il suo significato. Ė proprio la sua «polisemia» che crea la sua risonanza e la rende un' icona perfetta dell'ultimo "paesaggio» del poema.

Il tono estatico cambia negli ultimi versi del canto quando Beatrice parla del "gran seggio" che aspetta l'imperatore Arrigo VII e la vendetta di Dio contro il papa Clemente V che l'ha tradito. Quest'attacco contro l'ingannevole papa, con le sue allusioni a Bonifacio VIII e alla bolgia dei papi simoniaci, interrompe l'atmosfera celestiale che pervade il canto. A proposito di questo feroce attacco, Charles Singleton, il grande dantista americano, nel suo commento domanda, «Non ha imparato il pellegrino nessuna carità Cristiana?» ${ }^{4} \mathrm{E}$ Singleton non è stato l'unico a turbarsi dinanzi all'eruzione di rabbia e al ricordo dell'inferno in un momento paradisiaco. È anche sconvolgente quando ci rendiamo conto più tardi che queste sono le ultime parole di Beatrice.

I primi versi del canto trentuno ristabiliscono l'umore brevemente interrotto: «In forma dunque di candida rosa mi si mostrava la milizia santa/ che nel suo sangue Cristo fece sposa». Il «dunque» della prima riga implica la continuità di quello che seguirà con quello che è già stato detto. Dante amplifica la descrizione iniziale della candida rosa, ritornando alle due categorie, i beati e gli angeli, che sono apparsi come fiori e faville quando egli è entrato nell'Empireo. «La milizia santa/che nel suo sangue Cristo fece sposa» descrive i beati nel linguaggio del Libro degli Atti degli Apostoli (20.28) e nella metafora dello sposalizio mistico. Poi il testo si concentra sull'altra schiera, gli angeli, e sul ruolo di mediazione che essi svolgono nell'Empireo. Paragonandoli alle api che volano fra i beati e Dio, infiorandosi fra i beati e portando il nettare dall'alveare divino a loro. Il paragone di angeli e api era già in Sant'Anselmo, e molti commentatori vedono anche una riscrittura del passo del sesto libro dell'Eneide dove Enea vede le ombre nell'Eliso come una moltitudine di api che si posano sui fiori. La versione dantesca, però, è più attiva e bidirezionale, perché gli angeli vengono e vanno su e giù fra i fiori e l'alveare, fra le anime e Dio. ${ }^{5}$ Portando il nettare dall'alveare ai fiori invertono la direzione abituale della loro attività. Quel nettare è la quasi paradossale combinazione di pace e ardore che hanno acquisito dall'esperienza di Dio. Le api bidirezionali sono un esempio di un fenomeno naturale che è allo stesso tempo "fuori natura», come direbbe il poeta Yeats.

4. Charles S. Singleton, The Divine Comedy, Commento a Paradiso, Princeton: Princeton University Press, 1975, p. 510: «Has the wayfarer learned no lesson of Christian charity in the long journey to God, and does he, being now so near to God, not love his fellow man, not forgive?».

5. Cf. la discussione nel Commento a Paradiso di Robert Hollander, New York: Doubleday, 2006, p. 775. 
La qualità pittorica della descrizione dei bianchissimi angeli con i loro volti di «fiamma viva» e le loro ali d'oro ricorda gli angeli della pittura trecentesca, aggiungendo colore e movimento alla scena. La loro "plenitudine volante» non impedisce la vista e lo splendore della "luce divina", una luce "che è penetrante per l'universo secondo ch'è degno». [Questa frase ricorda le prime parole del Paradiso: «La Gloria di colui che tutto move/per l'universo penetra» e così via].

Il movimento dell'apertura del canto si conclude con una visione dell'unità dei beati di questo «sicuro e gaudioso regno", tutti concentrati sulla "trina luce» e scintillanti in "un unica stella» che simboleggia il divino. Per un momento Dante passa dal cielo alla terra, pregando, in un linguaggio che ricorda Boezio, che la «trina luce» guardi "qua giuso a la nostra procella», alla tempesta della vita terrena. Anche in questo momento di sublimità, il poeta rimane legato alla terra, «qua giuso».

Dopo le dieci terzine d'apertura, il testo si concentra sulla reazione di Dante alla scena che ha descritto, allo stupore e alla gioia che si sente all'arrivo nell'Empireo. Il canto include tre paragoni che comportano l'idea del pellegrinaggio e del compimento di una meta. Il primo comincia con la figura dei barbari, che, venendo dal nord, vedono Roma per la prima volta.

Se i barbari, venendo da tal plaga che ciascun giorno d'Elice si cuopra, rotando col suo figlio ond'ella è vaga, veggendo Roma e l'ardüa sua opra, stupefaciensi, quando Laterano a le cose mortali andò di sopra; io, che al divino dall'umano, all'etterno dal tempo era venuto, e di Fiorenza in popol giusto e sano, di che stupor dovea esser compiuto!

Per Dante è Roma anziché Gerusalemme che serve come modello privigilato del cielo, "quella Roma onde Cristo è romano" (Purg. XXXII.102). Dante identifica Roma con la sua ardua opera, in particolare il Laterano. Il Palazzo Laterano era stato donato da Costantino a papa Silvestro, e serviva da residenza papale fino alla partenza del Papato per Avignone nel 1309. La grande basilica era stata fondata da Costantino, e tutto il complesso di palazzo e basilica era un centro papale nel medioevo. Fu lì, nella sua loggia della benedizione, che Bonifacio VIII proclamò il giubileo del 1300. Per Dante il Laterano poteva rappresentare la gloria della Roma imperiale e papale. Però si deve tener conto dell'opinione del poeta sulle consequenze disastrose della «Donazione di Costantino». La Donazione era un documento che raccontava la storia della conversione dell' Imperatore e il suo dono alla Chiesa. Malato di lebbra, Costantino sarebbe guarito dopo essere stato battezzato da papa Silvestro. Dopo la sua conversione, egli avrebbe dato al papato i territori di Roma e il potere temporale. Il documento era un falso, come ha provato Lorenzo Valla nel Quattrocento. Dante pensava che fosse vero, ma lo considerava illegale e 
sbagliato perché aveva dato alla Chiesa il potere temporale. Feroce critico del papato del suo tempo, nondimeno Dante ammirava la grandezza del Laterano. Quando Dante scriveva del Laterano, sapeva che era già stato quasi distrutto da un incendio nel 1308, e non serviva più da residenza papale. (Dopo il ritorno da Avignone, i papi andarono a vivere in Vaticano).

Nel grande paragone dei barbari Dante dice che essi vengono «da tal plaga che ciascun giorno d'Elice si cuopra/rotando col suo figlio ond'ella è vaga». Vengono, cioè, dal Nord, dalla regione dove le costellazioni dell'Orsa Maggiore e dell'Orsa Minore sono sempre visibili. Dante combina l'astronomia e la mitologia, alludendo a Ovidio che racconta come Elice, anche chiamata Callisto, una delle ninfe seguaci di Diana, dea della castità, fosse stata presa da Giove e rimasta incinta. Cacciata da Diana per aver perso la sua verginità, Elice/Callisto era stata poi mutata in orsa dalla gelosa Giunone, moglie di Giove. Quando stava per essere uccisa da suo figlio Arcas, tutti e due, madre e figlio, furono trasformati da Giove nelle costellazioni dell'Orsa Maggiore e Minore. ${ }^{6}$

Credo che qui Dante abbia riveduto la figura di Elice di cui ha scritto nel venticinquesimo del Purgatorio lodando Diana come emblema di castità. Elice sembra colpevole quando Dante dice, «Al bosco/si tenne Diana, ed Elice caccionne/che di Venere aveva sentito il tosco». Ma la ninfa non era colpevole, perché era stata presa con la forza da Giove. La piccola frase, "ond'ella è vaga» dà enfasi alla continuità dell'amore materno, un soggetto sempre trattato con tenerezza da Dante.

Con il paragone dei barbari davanti alle meraviglie di Roma Dante introduce tre antitesi che, come dice John Scott, creano un crescendo di opposti. ${ }^{7}$ L'entrata nell' Empireo è racchiusa nel movimento «al divino dall' umano, all'eternità dal tempo, "—e poi, in un'inversione chiastica», da Firenze a un popolo giusto e sano». Com'è amaro quest'accenno a Firenze, l'ultimo del poema. Dante, così vicino alla fine, menziona i grandi temi di tutto il poema: l'umano e il divino, il tempo e l'eternità, Firenze e il cielo.

Il paragone dei barbari che arrivano a Roma è la prima delle tre similitudini di questo canto che utilizzano l'idea di pellegrinaggio. Ciascuno di questi paragoni comporta uno spazio più limitato e un'emozione più forte. Il secondo è anche più vicino alla situazione attuale di Dante:

E quasi peregrin che si ricrea nel tempio del suo voto riguardando, e spera già ridir com'ella stea, su per la viva luce passeggiando, menava io li occhi per li gradi, mo su, mo giù e mo recirculando.

6. Metamorphoses, II. 409-507.

7. John A. Scott, Understanding Dante, Notre Dame, Indiana: University of Notre Dame Press, 2004, p. 207. Cf. la sua lettura di questo canto in Lectura Dantis Turicensis: Paradiso, ed. Georges Güntert e Michelangelo Picone, Firenze: Cesati, 2002, p. 473-89. 
Qui è esplicito il senso d'arrivo a una meta desiderata per lungo tempo. Il Pellegrino che spera di «ridir» quello che vede è il poeta che noi leggiamo. In questo momento Dante personaggio e Dante poeta sono la stessa cosa.

Cosa vede Dante quando guarda intorno? Vede i visi e i corpi dei beati:

Vedea visi a carità süadi,

d'altrui lume fregiati e di suo riso,

e atti ornate di tutte onestadi.

Questa vista introduce, come dice la Chiavacci Leonardi, i primi veri corpi della Commedia. I "corpi fittizi» dei morti, per quanto siano vivaci e vividi, erano in realtà solo ombre. Nel Paradiso, fino all' Empireo, i beati non hanno neanche la forma umana; appaiono come luci, come gioelli, come fiamme sempiterne. Solo ora Dante vede i beati come saranno dopo l'ultimo giudizio, cioè nei corpi gloriosi. Il tema del desiderio dei morti per i loro corpi è un topos teologico, ma è espresso da Dante con particolare passione. Pensiamo al discorso di Solomone nel quattordicesimo canto del Paradiso sulla revestita "carne gloriosa e santa», e alla risposta dei beati alle sue parole:

Tanto mi parver subiti e accorti

l'uno e l'altro coro a dicer «Amme!»

che ben mostrar disio d'i corpi morti:

forse non pur per lor, ma per le mamme,

per li padri e per li altri che fuor cari

anzi che fosser sempiterne fiamme.

Pensiamo anche alla richiesta di Dante di vedere San Benedetto con «l'imagine scoverta", cioè, nel corpo, e poi al suo tentativo fallito di vedere il corpo di San Giovanni che qualche leggenda dice sia asceso nella carne. Dante nega la leggenda dell'ascensione di San Giovanni, ma è interessante che essa sia stata dipinta da Giotto nella cappella Peruzzi in Santa Croce a Firenze parecchi anni dopo l'esilio di Dante. ${ }^{8}$

L'affermazione di Dante secondo la quale egli vede i beati come saranno alla fine del tempo è non soltanto eterodossa, ma anche problematica entro i termini del suo mondo poetico. Poiché Dante dichiara che l'anfiteatro dei beati non è ancora completo, si potrebbe dire che allo stesso tempo egli lo vede e non lo vede come sarà alla fine del tempo. Qui abbiamo uno dei paradossi del paradiso dantesco: al protagonista è concessa una veduta di ciò che non è ancora successo.

Il ritorno del corpo nella sua specificità storica — giovane e vecchio, femmina e maschio - al punto più etereo del poema mi sembra un segno potente delle intuizioni e dei desideri del poeta. Ci ricordiamo, allora, Paradiso XXV, dove Dante parla della sua speranza più grande, quella della Resurrezione:

8. Cf. il mio saggio, "Dante and the Legend(s) of St. John», in Dante Studies CXVII (1999), p. 45-58. 
...Le nove e le scritture antiche pongon lo segno ed esso lo mi addita, de l'anime che Dio s'ha fatte amiche.

Dice Isaia che ciascuna vestita ne la sua terra fia di doppia vesta, e la sua terra è questa dolce vita; e 'l tuo fratello assai vie più digesta, là dove tratta de le bianche stole, questa revelazion ci manifesta.

Le bianche stole di cui scrive San Giovanni sono quelle che ora Dante vede nella rosa sempiterna.

In questo passo di alta serenità Dante si gira verso Beatrice, — ma non la trova. E uno shock per lui, e anche per il lettore. Non c'è stata nessuna preparazione per la sostituzione di Beatrice. Anzi, nel primo canto dell' Inferno Virgilio aveva parlato solo di "un' anima più di me degna» che sarebbe stata la guida di Dante «a le beate genti». Quando Dante si gira verso Beatrice per fare delle domande, trova un altro:

Uno intendea, ed altro mi rispuose:

credea veder Beatrice e vidi un sene

vestito con le genti gloriose.

Diffuso era per li occhi e per le gene

di benigna letizia, in atto pio

quale a tenero padre si convene.

Pensiamo al momento simile nel trentesimo del Purgatorio quando Dante, girandosi verso Virgilio, scopre che è sparito, e piange. Il forte pathos di quella scena, forse la più commovente di tutta la Commedia, contrasta con l'umore dell' episodio nel nostro canto. Beatrice non è sparita, ma invece è tornata al suo trono nel terzo grado dalla cima della rosa, il posto dal quale era discesa a chiedere l'aiuto di Virgilio.

Le prime parole di Dante sono, «Ov’è ella?» La nuova guida spiega che egli è stato mandato da Beatrice, come Virgilio aveva dichiarato nel secondo canto dell'Inferno. Questo nuovo tenero padre assume il suo ruolo nella serie dei mediatori che assistono il viaggio. Dante trattiene il suo nome fino a più tardi, così mettendo l'enfasi su Beatrice, alla quale egli ora rivolge una preghiera:

O donna in cui la mia speranza vige,

e che soffristi per la mia salute

in inferno lasciar le tue vestige,

di tante cose quant' I'ho vedute,

dal tuo podere e da la tua bontate

riconosco la grazia e la virtute.

Tu m' hai di servo tratto a libertate

per tutte quelle vie, per tutt' i modi

che di ciò fare avei la potestate.

La tua magnificenza in me custodi, 
sì che l'anima mia, che fatt' hai sana, piacente a te del corpo disnodi.

La bellissima preghiera è il culmine della «poesia della lode» di Beatrice che aveva avuto inizio nella Vita nova. Beatrice risponde alla preghiera senza parlare; lei, che ha parlato tanto nel Paradiso, qui rimane silenziosa, e lo ricompensa con un sorriso prima di ritornare all' "etterna fontana».

Borges, in uno dei «Nueve essayos dantescos» intitolato «La última sonrisa de Beatriz», parla di questi versi come «i versi più commoventi nella letteratura», interpretandoli come il fallimento tragico di ricatturare la donna adorata. Scrive Borges che nessuno ha veramente capito la tristezza di questi versi. Ma questa lettura dice più di Borges che di Dante, perché per Dante questo non è affatto un momento tragico. Beatrice aveva definito i desideri di Dante per lei come quelli che menavano «ad amar lo bene di là dal qual non è che s'aspiri» (Purg. XXXI.24). Beatrice non può essere la meta finale perché l'amore per lei deve portarlo oltre lei stessa a Dio. Dante volge Beatrice verso Dio, «l'etterna fontana», perché fra poco Dante stesso guarderà la medesima fontana. Come dice Lino Pertile, «Beatrice deve scomparire non perché ella ama Dio più che Dante, ma perché Dante deve amare Dio più che Beatrice».?

La preghiera a Beatrice esprime la gratitudine per tutto ciò che ella ha fatto per lui, portandolo «da servo alla libertate». Dandole del tu per la prima volta, Dante prega Beatrice come se fosse una santa. L'uso del tu implica anche un affetto, quasi un' intimità non visibile fino ad ora. Questa preghiera anticipa e somiglia alla preghiera alla Vergine nell' ultimo canto. Le due figure femminili, Beatrice e Maria, sono state legate da Dante fin dalla Vita nova, dove egli pensa alla sua donna dopo la sua morte «sotto la insegna di quella regina benedetta virgo Maria, lo cui nome fue in grandissima reverenza ne le parole di questa Beatrice beata». Nel secondo canto dell' Inferno abbiamo imparato che Maria iniziò il soccorso di Dante e che Beatrice venne nel Limbo per chiedere l'aiuto di Virgilio. Dante richiama quell' episodio quando dice che ella ha lasciato le sue vestige in Inferno per la sua "salute». Già nella Vita nova Beatrice era la Donna della Salute. Beatrice lasciando le sue vestige nell' Inferno ricorda anche Cristo che è sceso nell' Inferno per liberare i beati. Fra i tanti suoi ruoli Beatrice ricopre anche quello di figura Cristi. Come dice Charles Williams, Beatrice è «the Christ event» nella vita di Dante. ${ }^{10}$

La preghiera è piena di sostantivi astratti: podere, bontate, virtute, potestate, magnificenza. La bellezza di Beatrice, tema centrale del poema, non è menzionata qui, e Dante non parla neanche del suo amore per lei. Un' altra cosa da notare è la distanza fra Beatrice e Dante, una distanza grande come si poteva immaginare, ma anche irrilevante visto che ella è totalmente visi-

9. Lino Pertile, «Does the Stilnovo Go to Heaven?» in Dante for the New Millennium, ed. Teodolinda Barolini e H. Wayne Storey, New York: Fordham University Press, 2003, p. 111.

10. Charles Williams, The Figure of Beatrice: A Study in Dante, New York: The Noonday Press, 1961). 
bile a Dante. Nel mondo fuori dello spazio e del tempo la legge naturale non funziona.

Presso e lontano, lì, né pon non né leva:

ché dove Dio sanza mezzo governa

la legge natural nulla rileva (Par. XXX, 121-23).

Quanto tutta la "plenitudine volante» degli angeli non impedisce la diffusione della luce divina, tanto lo spazio vasto tra Beatrice e Dante non impedisce la visibilità della sua effige. Questa parola «effige» è importante perché è usata solo un' altra volta, negli ultimi versi del poema quando Dante cerca di vedere la «nostra effige» dentro i cerchi della visione finale.

Di solito la sostituzione di San Bernardo a Beatrice viene discussa in termini del perché questa scelta sia opportuna. Certo Bernardo è uno dei personaggi più affascinanti del dodicesimo secolo, famoso come mistico e anche come uomo attivissimo: fra l'altro era stato consulente di papi e di re, predicatore della seconda crociata, teologo polemico, autore di riforma monastica, predicatore eloquente, e scrittore prolifico di trattati e sermoni. Bernardo aveva tante qualità e tanti talenti che Dante avrebbe ammirato, ma il poeta si sofferma solo sulla sua esperienza contemplativa e sulla sua devozione mariana.

Bernardo, scrive Dante, «contemplando, gustò di questa pace». Il Bernardo storico aveva negato la possibilità di una visione di Dio diretta, faccia a faccia, in questa vita, ma il Bernardo del poema sta per pregare che Dante abbia quest'esperienza. Joseph Mazzeo nota che Dante non dice di San Bernardo che aveva visto il cielo in questa vita, ma solo che gustò della sua pace. ${ }^{11} \mathrm{Il}$ ruolo di Bernardo, dunque, è un po' problematico, o forse provocatorio, visto che egli chiede per Dante un'esperienza che, in vita, non aveva creduto possibile.

La vera domanda, però, è perché Dante scelga qualcuno per assumere il ruolo che Beatrice ha avuto fino ad ora. Parecchi commentatori, seguendo Pietro di Dante, dicono che Bernardo prende il posto di Beatrice perché, per attingere alla visionr beatifica, la contemplazione deve superare la teologia. Ma questo mi sembra troppo semplicistico. Più interessante è l'idea di Lino Pertile che Dante forse aveva pianificato il ritorno di Santa Lucia a questo punto, data la sua importanza come una delle "tre donne benedette " di Inferno II e il suo ruolo nel condurre Dante all'angelo della porta del Purgatorio. Secondo Pertile, Dante ha cambiato idea mentre scriveva e non ha voluto mantenere l'atmosfera stilnovistica nella quale la santa era presentata. ${ }^{12}$ Per me, sembra più giusto che San Bernardo, il «dottor mellifluis» della tradizione, gran maestro retorico, faccia la preghiera magnifica alla Vergine. Bernardo rappresenta anche un ritorno al mondo dei padri; Dante ha avuto una serie di padri, Virgilio, Brunetto, e Cacciaguida per nominarne tre-. Bernardo è l'unico perso-

11. Joseph Anthony Mazzeo, Structure and Thought in the "Paradiso", Ithaca, NY: Cornell University Press, 1958, p. 90-91, 99. E cf. il saggio già citato di Hawkins, p. 185.

12. Pertile, p. 11-13. 
naggio per cui l'aggettivo "tenero» è usato, e sembra quasi un padre materno, come a volte è Virgilio.

Un altro aspetto interessante della presentazione di Bernardo è la sua vecchiaia. Due volte Dante si referisce a lui con il latinismo «sene», dal latino «senex». Così Dante segna la sua partenza dalla tradizione teologica in cui Sant'Agostino, San Tommaso e quasi tutti i Padri e i Dottori della Chiesa sostengono che la Resurrezione avrà luogo nell' "età perfetta", cioè verso i trent'anni, quando morì Cristo. I beati di Dante comprendono non soltanto $i$ vecchi ma anche $\mathrm{i}$ bambini che vedrà nel canto seguente. L'enfasi sull'età di Bernardo, come i latinismi, fanno crescere il senso della sua dignità.

Bernardo si nomina solo dopo la preghiera a Beatrice, e lo fa in un linguaggio cortese:

E la regina del cielo, ond'ío ardo tutto d'amor, ne farà ogne grazia, però ch'i' sono il suo fedel Bernardo.

Dante risponde alla scoperta della sua identità con grande emozione. Qui abbiamo il terzo e l'ultimo accenno al pellegrinaggio. Dante si paragona a un pellegrino che viene da lontano per vedere la Veronica, il velo o il panno che si pensava portasse impresso il volto di Cristo.

Qual è colui che forse di Croazia

viene a veder la Veronica nostra

che per l'antica fame non sen sazia,

ma dice nel pensier, fin che si mostra;

«Segnor mio Iesù Cristo, Dio verace,

or fu sì fatta la sembianza vostra?»

Diverse leggende si sono combinate per creare la storia di una donna di Gerusalemme che ha dato un panno, un «sudarium», a Cristo mentre saliva il Calvario. Dopo questo atto compassionevole l'immagine del suo volto è rimasta sul panno. Il nome Veronica, combinando le parole "vera»e «icona», fu legato alla donna e diventò un aspetto della leggenda.

La Veronica era la reliquia più preziosa e più famosa del medioevo. Autenticata da Papa Innocenzo III nel 1216, veniva mostrata nella basilica di San Pietro il venerdì e nei giorni santi, ed era collegata alla concessione di indulgenze. La Cronica di Giovanni Villani nota che l'ostensione era avvenuta diverse volte durante il Giubileo del 1300, — quando forse Dante stesso avrebbe potuto assistervi. Ma Dante sa della Veronica già dal tempo della Vita nova, perché verso la fine del libello scrive di un gruppo di pellegrini che muovono verso Roma. Pensa che essi vadano proprio per vedere «quella immagine benedetta la quale Gesù Cristo lasciò a noi per esempio de la sua bellissima figura, la quale vede la mia donna gloriosamente». Sono qui collocate le idee di pellegrinaggio, della Veronica, e di Beatrice «in gloria» che lo «spirito peregrino» di Dante intravede e di cui le ultime parole della Vita nova parlano. Tutti questi elementi sono presenti di nuovo nel trentunesimo canto, dove Dante veramente vede 
la sua donna «in gloria». Questa riscrittura della fine della Vita nova verso la fine della Commedia crea un bel senso di coerenza, unificando il libello giovanile con l'opera dei suoi ultimi anni e chiudendo il circolo.

Il paragone del volto di Cristo con quello di San Bernardo è sembrato inadatto a parecchi lettori. Il commento di Bosco e Reggio spiega che esso non riguarda due visi, ma piuttosto l'intensità di desiderio nel pellegrino croato e in Dante. "Qualcuno obietta», scrivono, "che è sconveniente paragonare la commozione di vedere le fattezze di Bernardo a quella di vedere il volto di Gesù; ma il paragone non è di due volti, bensì tra due desiderii e tra due moti quasi d'incredulità per esser giunto a soddisffarlo». ${ }^{13} \mathrm{Mi}$ domando, però, se Dante abbia davvero avuto tanta voglia di vedere il volto di San Bernardo, e... ho i miei dubbi.

Per me è più importante invocare la Veronica per creare il legame con la Vita nova di cui ho parlato. Poi, il desiderio di vedere il viso di Cristo suggerisce anche la conclusione della Commedia, dove Dante cerca di vedere il volto di Cristo, «la nostra effige» nella visione finale. Dietro le parole sulla Veronica c'è il forte bisogno di incontrare il vero Cristo che informa gli ultimi versi del poema.

Fino a questo punto Dante ha guardato la candida rosa nella sua «forma generale». Ora Bernardo lo dirige a focalizzare sul punto più luminoso e più alto, sulla regina del regno. Maria è paragonata all'aurora, com'è stata Beatrice quando è apparsa nel paradiso terrestre alla fine del Purgatorio. Circondata dagli angeli che cantano e giocano intorno a lei, Maria somiglia alla figura di una Maestà, una Madonna regale, anche se non accompagnata dal Bambino. Dante la chiama "pacifica oriafiamma", una formula di ossimoro poiché l'oriafiamma era lo stendardo dei re francesi, segno di guerra anziché di pace com'è qui. Intorno a Maria tutto è letizia e delizia. Il canto si conclude com'è cominciato, parlando degli angeli, qui in rapporto a Maria invece che ai beati.

vid'io più di mille angeli festanti, ciascun distinto di fulgore e d'arte.

Vidi a lor giochi quivi e a lor canti ridere una bellezza, che letizia era ne li occhi a tutti li altri santi.

Bernardo dirige Dante verso Maria perché è solo lei che può aiutarlo ad avere la visione beatifica. Maria, ricordiamo, ha messo in moto il soccorso di Dante dalla selva oscura. E' stata lei a inviare Santa Lucia da Beatrice nella catena di grazia descritta nel secondo canto dell' Inferno.

Maria è raffigurata su ciascuna delle terrazze del Purgatorio come esempio delle virtù che $\mathrm{i}$ penitenti cercano di acquistare. Era già stata presente in una visione preliminare nel canto XXIII, dove sono rappresentate simbolicamente l'Annunciazione, l'Assunzione, e l'Incoronazione. Qui nel canto XXXI l'ac-

13. Umberto Bosco e Giovanni Reggio, Commento a Paradiso, Firenze: Le Monnier, 1982, p. 506 (Introduzione al canto XXXI). 
cento è sulla sua regalità e la sua bellezza. Ora che Beatrice non è più presente, il linguaggio della pulcritudine può essere diretto a Maria. Nel canto seguente l'attenzione su di lei, «la faccia che a Cristo più si somiglia», sarà anche più forte. Dante deve avvicinarsi a Maria per ottenere la grazia per cui finalmente si avvicinerà a Dio.

Alla fine del canto tutti e due, Dante e Bernardo, stanno ardentemente mirando la Vergine. Sembra che siano pronti per pregarla,_ - ma c'è un altro canto che interviene fra questo momento e le parole che Bernardo indirizzerà a Maria. Questi due canti finiscono quasi nello stesso modo, concentrandosi sulla figura della Madonna. Come dirà Bernardo, «chi vuol grazia e a te non ricorra, sua disïanza vuol volar sanz'ali». Nel canto XXXII Dante sarà chiamato da Bernardo "figliuol di grazia», e sarà preparato per quella grazia che lo porterà «all'ultima salute».

Vorrei concludere sottolineando l'importanza dell'idea di pellegrinaggio in questo canto. Nell' Inferno, dove la forma del suo viaggio era una discesa nel basso aldilà, Dante non parla mai del pellegrinaggio. La parola entra nel lessico del poema solo nel Purgatorio. Ora che sta per terminare, il viaggio si rivela come un pellegrinaggio che ha per suo fine la visio Dei ed anche il poema che stiamo leggendo. L'anno di questo viaggio, il 1300, era un anno di grande pellegrinaggio a Roma, essendo l'anno del Giubileo proclamato da Bonifacio VIII, il papa così vilificato da Dante. Vorrei suggerire che Dante ha creato nel suo poema un'alternativa personale al Giubileo ufficiale. Il Laterano e la Veronica, sito e reliquia fondamentale nella Roma papale del 1300, si trovano ora «in quella Roma onde Cristo è romano:» il paradiso immaginato e creato da Dante. $^{14}$

14. Vorrei ringraziare Emma Mallardi e Piero Boitani per il generoso aiuto che mi hanno dato nella preparazione di questo saggio. 